\title{
Prevenção e tratamento do hiperparatireoidismo secundário na DRC
}

\author{
Prevention and treatment of secondary hyperparathyroidism in CKD
}

Autores:

José Edevanilson

Barros Gueiros

Fabiana Rodrigues

Hernandes

Cristina Karohl

Vanda Jorgetti
1 Avaliação dos níveis de paratormônio (PTH) e fosfatase alcalina (FA) na DRC

1.1 Os níveis séricos de PTH devem ser analisados em todos os pacientes com DRC, cuja taxa de filtração glomerular (TFG) for inferior a $60 \mathrm{~mL} / \mathrm{min} / 1,73 \mathrm{~m}^{2}$ (Evidência). A Tabela 1 descreve as frequências recomendadas (Opinião).

\begin{tabular}{|c|c|c|}
\hline TABELA 1 & \multicolumn{2}{|c|}{$\begin{array}{l}\text { FREQUÊNCIA DE AVALIAÇÃO } \\
\text { DOS NÍVEIS DE PTH NOS } \\
\text { DIFERENTES ESTÁGIOS DA } \\
\text { DRC }\end{array}$} \\
\hline $\begin{array}{c}\text { Estágio da } \\
\text { DRC }\end{array}$ & $\begin{array}{c}\text { TFG } \\
\text { (mL/min) }\end{array}$ & $\begin{array}{c}\text { Frequência } \\
\text { de coleta }\end{array}$ \\
\hline III & $30-59$ & Anual \\
\hline IV & $15-29$ & Semestral \\
\hline V ou VD & $\begin{array}{l}<15 \text { ou } \\
\text { diálise }\end{array}$ & Trimestral \\
\hline
\end{tabular}

1.2 Deve-se modificar a frequência de avaliação dos níveis séricos de PTH se os resultados das análises mostrarem uma tendência de elevação ou de descenso ou após a instituição do tratamento, seja ele para reduzir ou elevar os níveis de PTH (Opinião).

1.3 A amostra de sangue para dosagem do PTH deverá ser obtida, preferencialmente em jejum, nos pacientes com DRC em tratamento conservador ou naqueles em diálise peritoneal (DP). Nos pacientes em hemodiálise (HD), as amostras poderão ser obtidas no início da sessão de diálise (Opinião).

Os laboratórios de análises clínicas devem informar ao médico qual o método que utilizam para a dosagem do PTH e, para cada método, qual a fonte ideal: plasma ou soro, além das características ideais de coleta e armazenamento (Evidência).

1.5 Nos pacientes com DRC estágios IV e $\mathrm{V}$ em tratamento conservador ou diálise, a FA deve ser dosada anualmente, ou mais frequentemente na vigência de níveis elevados de PTH (Evidência).

2 Prevenção e tratamento do hiperparatireoidismo secundário na DRC

\section{Pacientes com DRC estágios III e IV}

2.1 Nos pacientes com DRC estágios III a V, os níveis ideais de PTH não são conhecidos. No entanto, sugerimos que os pacientes com níveis de PTH acima do limite superior de referência para o método sejam avaliados quanto à presença de hipocalcemia, hiperfosfatemia ou fração de excreção de P elevada, e deficiência de 25-hidroxivitamina D (25-vit D). Se forem detectadas alterações nesses parâmetros, estes devem ser corrigidos; sais de Ca para correção da hipocalcemia, orientação dietética e/ou uso de quelantes de $\mathrm{P}$ para correção da hiperfosfatemia e uso de ergocalciferol (vitamina D2) ou colecalciferol (vitamina D3) para correção da hipovitaminose D (Evidência).

2.2 Se os níveis de 25-vit D forem inferiores a $30 \mathrm{ng} / \mathrm{mL}$, inicia-se a suplementação com vitamina $\mathrm{D}_{2}$ ou vitamina $\mathrm{D}_{3}$ (Tabela 2) (Opinião). 
Tabela 2 Suplementação com ergocalciferol (Vitamina D2) ou colecalcifrol (Vitamina D3) com base nos NÍVEIS SÉRICOS DE 25-VIT D

\begin{tabular}{ccc} 
Nível sérico $(\mathrm{ng} / \mathrm{mL})$ & Dose de ergocalciferol ou colecalcifrol & Tempo de suplementação \\
\hline$<5$ & $50.000 U \mathrm{Ul} / \mathrm{sem} / 12 \mathrm{sem}$. Após, $50.000 \mathrm{UI} / \mathrm{mês}$ & 6 meses e repetir dosagem 25-vit D \\
$5-15$ & $50.000 \mathrm{UI} / \mathrm{sem} / 4 \mathrm{sem}$. Após, $50.000 \mathrm{Ul} / \mathrm{mês}$ & 6 meses e repetir dosagem 25-vit D \\
$16-30$ & $50000 \mathrm{UI} / \mathrm{mês}$ & 6 meses e repetir dosagem 25-vit D \\
\hline
\end{tabular}

2.2.1 Durante a suplementação com vitamina $\mathrm{D}_{2}$ ou $\mathrm{D}_{3}$, os níveis séricos de cálcio $(\mathrm{Ca})$ e $\mathrm{P}$ devem ser analisados a cada 2 meses (Opinião).

2.2.2 Se os níveis séricos de Ca forem superiores aos limites de referência do método, suspender a suplementação (Evidência).

2.2.3 Se o P sérico for superior ao valor de referência, inicia-se ou ajusta-se a dose de quelantes de P. Se após essa conduta a fosfatemia persistir elevada, suspender a suplementação com vitamina $\mathrm{D}_{2}$ ou $\mathrm{D}_{3}$ (Opinião).

2.3 Se os níveis séricos de PTH permanecerem elevados apesar da correção dos fatores descritos anteriormente, indica-se o tratamento com calcitriol ou análogo da vitamina D. Recomenda-se o tratamento com calcitriol na dose de 0,25-0,5 mg/dia. Entretanto, o Ca e o P séricos devem permanecer dentro dos limites de referência, além de a função renal permanecer estável (Evidência).

\section{Pacientes com DRC estágio V ou V D}

2.4 Pacientes com DRC estágio V ou V D devem manter os níveis de PTH entre 2 e 9 vezes o valor superior do método. No entanto, se as avaliações mostrarem tendência de elevação ou redução nos níveis de PTH, medidas terapêuticas devem ser introduzidas e a frequência de dosagem aumentada (Evidência).

2.5 Pacientes com PTH elevado ou com tendência à elevação devem ser tratados com calcitriol ou análogos de vitamina D e/ou calcimiméticos (Evidência).

2.6 O critério de seleção para a escolha da droga deve levar em consideração o perfil bioquímico do Ca e P do paciente (Opinião):
2.6.1 Pacientes com hipercalcemia e/ou hiperfosfatemia, o uso de calcitriol ou de análogos da vitamina $\mathrm{D}$ devem ser evitados até a correção dos níveis de Ca e de P (Evidência).

2.6.2 Pacientes com hipocalcemia, o uso de calcimimético deve ser evitado até a correção dos níveis de Ca (Evidência).

2.7 Pacientes tratados com calcitriol ou análogos de vitamina D que desenvolvam hipercalcemia e/ou hiperfosfatemia devem ter a dose da medicação reduzida ou suspensa (Evidência).

2.8 Pacientes tratados com calcimiméticos que desenvolvam hipocalcemia devem ter a sua dose reduzida ou suspensa de acordo com a gravidade da hipocalcemia e quadro clínico apresentado pelo paciente (Evidência).

2.9 A escolha do tipo de quelante de P, bem como a sua dose, devem ser ajustadas de forma a permitir que níveis séricos de Ca e $\mathrm{P}$ mantenham-se dentro dos valores de referência, possibilitando o tratamento do hiperparatireoidismo secundário (HPS) (Opinião).

2.10 Pacientes que apresentam redução dos níveis de PTH abaixo de $150 \mathrm{pg} / \mathrm{mL}$ devem ter as doses de calcitriol ou análogos de vitamina D e/ou calcimiméticos reduzidas ou suspensão das drogas (Evidência).

2.11 O controle de tratamento deve ser feito por meio da dosagem mensal de PTH, Ca, P até que os níveis de PTH estejam estáveis dentro da faixa recomendada (Opinião).

2.12 Pacientes que, a despeito das medidas clínicas e terapêuticas adotadas, persistirem com níveis de PTH acima da faixa recomendada devem ser tratados com paratireoidectomia (Evidência). 


\section{RACIONAL}

Nos pacientes com DRC, os níveis séricos de PTH se elevam quando a TFG diminui abaixo de $60 \mathrm{~mL} /$ $\mathrm{min} / 1,73 \mathrm{~m}^{2} .{ }^{1}$ As alterações ósseas decorrentes do excesso de PTH podem estar presentes já no estágio III da DRC. Com a progressão da doença renal, estas alterações tornam-se mais evidentes. Dessa forma, a avaliação dos níveis séricos de PTH se inicia a partir do estágio III.

As técnicas empregadas para a determinação do PTH sérico são: imunorradiometria (IRMA) e imunoquimioluminescência (ICMA). Essas técnicas superestimam os níveis de PTH biologicamente ativos, pois detectam fragmentos não ativos da molécula ${ }^{2}$, o que explica a presença de altos níveis de PTH associados à doença óssea de baixa remodelação em alguns pacientes. $^{3-5}$ Novos ensaios para dosagem desse hormônio têm sido desenvolvidos, porém, até o presente momento, não existem estudos que demonstrem a superioridade desses ensaios, com relação aos anteriores, uma vez que não foram realizados estudos com biopsia óssea, para avaliar se distintas faixas de níveis de PTH são capazes de predizer que tipo de lesão óssea acomete o paciente. ${ }^{6-9}$

Vale lembrar que existem vários testes para dosagem de PTH. A maioria dos estudos realizados até a publicação do KDOQI 2003 baseou-se nos resultados com o teste produzido pela Nichols (Allegro) e que atualmente não é comercializado. ${ }^{10}$ Estudo publicado recentemente comparou resultados de dosagem do PTH empregando-se os testes disponíveis no mercado, com os resultados obtidos com o teste da Nichols/ Allegro. Os resultados mostraram uma variabilidade significante do resultado do nível de PTH nos diferentes testes, pois estes testes dosavam quantidades diferentes de moléculas ativas ou inativas do PTH. ${ }^{11-12}$

É de fundamental importância que as amostras colhidas, para a dosagem do PTH, sejam imediatamente colocadas em gelo, rapidamente centrifugadas em centrífuga refrigerada, evitando, assim, que a molécula se degrade, o que poderia interferir nos resultados. Importante também é ter conhecimento do método empregado para a dosagem bem como os valores de referência do laboratório.

A FA é uma enzima que retira o $\mathrm{P}$ das proteínas e dos nucleotídeos. Está presente em todo o organismo na forma de isoenzimas específicas para cada tecido. Altas concentrações de FA são encontradas no fígado e nos ossos, portanto, elevações na dosagem sérica desta enzima ocorrem por alterações hepáticas, alta remodelação óssea ou metástases ósseas. Os valores de FA também se encontram aumentados nos casos de crianças em fase de crescimento ósseo ou após fraturas. A dosagem da FA nos pacientes com DRC, desde que não apresentem alterações hepáticas, serve como um teste adjunto no diagnóstico do DMO subjacente e também como um parâmetro no acompanhamento do tratamento do HPS.

Nos pacientes com DRC estágios III a V (conservador), os níveis ideais de PTH não são conhecidos. Nos estágios iniciais da DRC, as alterações dos níveis de PTH representam a adaptação do organismo à perda da função renal, visando manter os níveis de $\mathrm{Ca}$ e $\mathrm{P}$ séricos adequados. Ainda não sabemos como distinguir uma resposta adaptativa de uma situação anormal. Portanto, a melhor conduta será aumentar a frequência de dosagem dos níveis de PTH se houver uma tendência à elevação desse hormônio e, nesse caso, iniciar o tratamento com calcitriol ou análogos da vitamina D após correção da hipocalcemia, da hiperfosfatemia ou da deficiência de vitamina 25 -vit D, se estiverem presentes.

A suplementação com Ca, nos pacientes com DRC nos estágios III a V (conservador), deve ser cautelosa e apenas nos casos diagnosticados de hipocalcemia. Estudos com população normal ${ }^{13}$ e pacientes com DRC em tratamento conservador ${ }^{14}$ já demonstraram um aumento do risco cardiovascular e da calcificação coronariana associados à suplementação de $\mathrm{Ca}$.

Devemos acompanhar os níveis de $\mathrm{P}$ séricos e naqueles pacientes com níveis elevados, iniciar dieta e uso de quelantes à base de Ca quando necessários.

Recentemente, demonstrou-se que pacientes em tratamento conservador e com níveis normais de $P$ podem apresentar elevação dos níveis séricos de PTH e do FGF-23, sugerindo uma sobrecarga de P. Portanto, a fosfatemia parece não ser um marcador ideal da carga de P no organismo, chamando atenção para a importância do controle do conteúdo de $P$ na dieta e da monitoração da fração de excreção de P em fases precoces da DRC. ${ }^{15}$

A 25 -vit D tem duas formas e inúmeros metabólitos. As duas formas são o ergocalciferol (vitamina $\mathrm{D}_{2}$ ) e o colecalciferol (vitamina $\mathrm{D}_{3}$ ) e seus principais metabólitos são o calcidiol ou 25-vit D e o calcitriol ou 1,25-vit D. O termo 25-hidroxivitamina D não deve ser confundido com calcitriol que, na verdade, se trata de um hormônio sintetizado principalmente nos rins, a partir da 25 -vit D.

Os níveis séricos de 25 -vit $\mathrm{D}$ representam o estoque corporal de vitamina $\mathrm{D}$. Indivíduos com níveis séricos inferiores a $15 \mathrm{ng} / \mathrm{mL}$ são classificados como deficientes em vitamina $\mathrm{D}$, e aqueles cujos níveis 
encontram-se entre 16 e $30 \mathrm{ng} / \mathrm{mL}$, como insuficientes. Estudos realizados nos Estados Unidos, Europa e inclusive no Brasil mostram prevalências de $20 \%$ a $90 \%$ de deficiência/insuficiência nas populações estudadas, independentemente da raça, faixa etária e sexo. ${ }^{16-22}$

Pacientes com DRC também apresentam elevada prevalência de deficiência deste hormônio. ${ }^{23,24}$ Alguns estudos mostram deficiência superior a $70 \%$ nos pacientes com DRC estágios III e IV. ${ }^{23-25}$ Nos pacientes em HD e DP, essa prevalência é superior a $90 \%{ }^{26-28}$ e, nos transplatados renais, superior a $70 \%{ }^{29}$ Tanto em indivíduos normais como nos pacientes com DRC, níveis reduzidos de 25 -vit $\mathrm{D}$ associam-se a aumento dos níveis de PTH. Na DRC, a deficiência dessa vitamina também está associada a progressão e gravidade do HPS. Nos indivíduos normais, a deficiência de 25 -vit D está associada à menor densidade mineral óssea e à maior taxa de fraturas, ${ }^{29,30}$ o que também foi observado nos pacientes em diálise..$^{27,31}$

Esses dados revelam que tanto a deficiência como a insuficiência de 25 -vit D são danosas aos pacientes com DRC, agravando o HPS e causando prejuízo à saúde dos ossos. As principais causas de deficiência/ insuficiência de 25 -vit D na DRC são a baixa exposição solar, baixa ingestão de alimentos ricos em vitamina $\mathrm{D}$ e a diminuição da síntese endógena de vitamina D na pele. Devemos lembrar que indivíduos idosos também sintetizam menos vitamina $\mathrm{D}$ e, portanto, têm a deficiência de 25 -vit D agravada quando na presença de DRC. Também indivíduos de cor de pele negra apresentam maior risco de deficiência/insuficiência de 25 -vit $\mathrm{D}$, pois a presença da melanina diminui a síntese cutânea da vitamina D. ${ }^{31,32}$

A prevenção e o tratamento da insuficiência/deficiência de vitamina $\mathrm{D}$ nos pacientes com $\mathrm{DRC}$ estágios 3 e 4 têm sido preconizados visando reduzir a frequência e a gravidade do HPS. ${ }^{33}$ Poucos são os estudos que avaliaram a efetividade da suplementação com vitamina $\mathrm{D}_{2}$ ou $\mathrm{D}_{3}$ na DRC. A suplementação com ergocalciferol reduziu os níveis de PTH nos pacientes com DRC estágio 3. ${ }^{34-36}$ Nos pacientes em HD ou DP, a reposição de ergocalciferol foi segura e efetiva para normalizar os níveis de 25-vit D, o mesmo não ocorrendo com os níveis de PTH. ${ }^{25-27}$ Até o momento, a efetividade da reposição de vitamina $\mathrm{D}_{2}$ ou $\mathrm{D}_{3}$ neste grupo de pacientes não está estabelecida. Deve-se ressaltar que o calcitriol não deve ser usado para tratar insuficiência /deficiência de 25-vit D.

No Brasil foi recentemente lançada uma preparação comercial de vitamina $\mathrm{D}_{3}$ (colecalciferol - Addera D3 ${ }^{\circledR}$ - 134 UI/gota) como único elemento da formulação, pois em geral, as apresentações estão associadas à vitamina $\mathrm{A}, \mathrm{Ca}$ ou associadas à polivitamínicos, porém em baixa concentração. Esta nova formulação muitas vezes não supre a necessidade de reposição de 25-vit D nos casos de deficiências mais graves. Recomenda-se, então, manipular na forma de gotas. A concentração mais preconizada é de 1.000 UI/gota. Vale ressaltar que essa vitamina deve ser armazenada em geladeira e protegida da luz.

Os níveis séricos ideais de PTH para pacientes com DRC, seja em tratamento conservador ou em diálise, continuam a desafiar os nefrologistas. Fatores ligados à metodologia empregada na dosagem do $\mathrm{PTH}$, a falta de correlação entre histologia óssea e valores intermediários de PTH dificultam a determinação de níveis ótimos desse hormônio. ${ }^{12,37}$ Estudos mostraram associação entre aumento da mortalidade e níveis reduzidos ou elevados de PTH nos pacientes em diálise. ${ }^{38-40}$ Dessa forma, deve-se evitar manter os pacientes com níveis extremos e daí a sugestão de valores entre 2 e 9 vezes o limite superior do método. O seguimento, ao longo do tempo, é imprescindível para a conduta terapêutica. Detectando-se tendência de aumento ou redução do intervalo recomendado, medidas imediatas devem ser adotadas para o retorno aos níveis sugeridos. $\mathrm{O}$ tratamento com calcitriol ou análogos de vitamina $\mathrm{D}$ e/ou calcimiméticos deve ser instituído. $\mathrm{O}$ critério de escolha da droga depende dos níveis séricos de Ca e P. Dessa forma, pacientes com hipercalcemia e/ou hiperfosfatemia não devem utilizar calcitriol ou análogos de vitamina $\mathrm{D}$, e pacientes com hipocalcemia não podem ser tratados com calcimiméticos.

Pacientes com DRC, especialmente em diálise, apresentam níveis reduzidos de calcitriol. ${ }^{41}$ Esse é um dos fatores responsáveis pelo desenvolvimento e progressão do HPS, tanto por reduzir a absorção intestinal de $\mathrm{Ca}$, levando à hipocalcemia, como por diminuir o controle da síntese e secreção de PTH. Além disso, na DRC, a expressão dos receptores da 25 -vit D está diminuída nas glândulas da paratireoide, limitando a ação do calcitriol na inibição do gene do PTH. ${ }^{42-44}$

Os principais efeitos adversos do tratamento com calcitriol são a maior absorção intestinal de Ca e P podendo produzir hipercalcemia, hiperfosfatemia e aumentar o produto $\mathrm{Ca} \times \mathrm{P} .{ }^{45}$ Além disso, o calcitriol pode reduzir a formação óssea e originar uma condição de doença óssea de baixa remodelação, a chamada doença óssea adinâmica. ${ }^{46,47}$ Por tais motivos, os níveis séricos de PTH, Ca e P devem ser monitorados no curso do tratamento com calcitriol e sua dose ajustada para manter os níveis recomendados desses elementos. 
Calcitriol pode ser administrado tanto nas formas diária por via oral ou intermitente (em pulso), via intravenosa ou oral, 2 a 3 vezes por semana. A administração intermitente parece ser mais efetiva para controlar os níveis de PTH do que a forma diária. ${ }^{48,49}$ No entanto, não há um consenso na literatura sobre a melhor via de administração, assim como a melhor dose e frequência. Nos pacientes com hiperparatireoidismo leve a moderado não há diferença entre o uso oral intermitente ou diário na redução dos níveis de PTH. ${ }^{10,11}$ Nas formas graves, recomenda-se o uso intermitente.

Não existe um consenso na literatura sobre o uso de calcitriol intermitente oral ou intravenoso. O K/ DOQI analisou quatro estudos clínicos controlados nos quais a administração intermitente intravenosa foi comparada com a oral e concluíram que a intravenosa foi mais efetiva na supressão dos níveis de PTH. ${ }^{48,52-54}$ No entanto, estes estudos apresentam diferenças metodológicas que limitam os resultados. Dois destes compararam tratamento oral diário com tratamento intravenoso intermitente (3x/semana), portanto, não é possível concluir que o oral intermitente é inferior ao intravenoso. ${ }^{48,51}$ Além disso, os níveis de PTH no início do estudo eram inferiores a 400 $\mathrm{pg} / \mathrm{mL}$, ou seja, formas mais leves de HPS.

Slatopolsky e cols. comparam formas oral e intravenosa intermitentes e demonstraram que a intravenosa foi mais efetiva. ${ }^{55}$ Outros estudos não observaram diferença entre as formas de administração..$^{56-58}$

No hiperparatireoidismo leve a moderado tanto a via oral como intravenosa são efetivas. Nas formas mais graves a terapia intravenosa é mais eficaz. ${ }^{59,60}$ No entanto, faltam na literatura estudos clínicos que definam a melhor forma de administrar calcitriol. ${ }^{19}$ Nos pacientes em hemodiálise recomenda-se o uso intravenoso quando a dose for superior a $1 \mathrm{mg}$. Para pacientes em DP ou em tratamento conservador recomenda-se o uso oral, preferencialmente à noite.

As doses iniciais de calcitriol devem ser ajustadas de acordo com os níveis de PTH e gravidade do HPS. $\mathrm{O}$ ajuste da dose deve ser realizado a cada quatro semanas, no mínimo, nos três primeiros meses, quando a queda do PTH pode ser observada e há o risco de supressão acentuada. Estudos prospectivos mostram que pacientes com hiperparatireoidismo severo, isto é, com níveis de PTH superiores a 600 pg/mL, precisam de doses mais elevadas de calcitriol. Além disso, o tempo de tratamento é mais prolongado para avaliar resposta - no mínimo de 12 a 24 semanas. ${ }^{61,62}$

Diversos fatores são associados à falta de resposta ao uso de calcitriol: o volume das glândulas paratireoides, a menor sensibilidade ao $\mathrm{Ca}$, a menor densidade de receptores da 25 -vit D ou o desenvolvimento de hipercalcemia e/ou hiperfosfatemia. 43,63-65

Estudos sugerem que os análogos da vitamina D controlam efetivamente os níveis de PTH com menor incidência de efeitos colaterais, como hiperfosfatemia e hipercalcemia. Dentro dessa classe de drogas, o paricalcitol é o composto mais estudado. Sprague e cols., ${ }^{66}$ em um estudo duplo-cego randomizado, estudaram a eficácia do calcitriol e paricalcitol em suprimir a produção de PTH. Esses autores mostraram que ambas as drogas, são eficazes, porém os pacientes tratados com paricalcitol controlaram mais rapidamente os níveis de PTH. Esse estudo revelou também que a incidência de hipercalcemia foi semelhante nos dois grupos; porém, nos pacientes tratados com paricalcitol, a hipercalcemia mantida foi menos frequente.

Quanto ao tratamento com calcimimético, o mesmo se baseia na sua ligação com o receptor de Ca presente nas células paratireoides de forma a mudar a sua conformação, possibilitando melhor interação do $\mathrm{Ca}$ com este receptor e proporcionando maior supressão da secreção e síntese de PTH. Moe e cols. ${ }^{67}$ em uma análise secundária de três estudos randomizados e controlados que compararam a eficácia da terapêuticapadrão (uso de quelantes de $\mathrm{P}$ associados a calcitriol ou análogos de vitamina D) contra um grupo de pacientes que utilizaram a terapêutica-padrão acrescida do calcimimético (cinacalcete), mostraram que o acréscimo do cinacalcete permitiu que maior proporção de pacientes atingissem níveis adequados de $\mathrm{Ca}, \mathrm{P}$ e PTH. Entretanto, muitos pacientes abandonaram os estudos, diminuindo, assim, sua relevância.

Até o presente momento, a maioria dos estudos falhou na identificação de uma droga ideal, ou seja, que seus efeitos contribuam para melhores desfechos clínicos (mortalidade, hospitalização, fratura, qualidade de vida etc.). Isso ocorre, pois a maioria dos estudos apresenta limitações metodológicas (número insuficiente de pacientes, perdas elevadas de seguimento, tempo de seguimento curto, análises secundárias pouco conclusivas ou insuficientes, estudos observacionais etc.). Diante do exposto, não podemos apontar, de forma definitiva, qual o melhor esquema terapêutico para o tratamento do HPS. No entanto, considerando sua fisiopatologia, entendemos que a abordagem deva incluir várias drogas.

Para aqueles pacientes que não respondem aos esquemas terapêuticos, ou seja, desenvolvem hiperparatireoidismo refratário, resta a paratireoidectomia. Entretanto o nível exato de PTH que determina esta intratabilidade ainda não foi definido. ${ }^{68}$ 


\section{ReferÊnCIAS}

1. Reichel H, Deibert B, Schmidt-Gayk H, Ritz E. Calcium metabolism in early chronic renal failure: implications for the pathogenesis of hyperparathyroidism. Nephrol Dial Transplant. 1991; 6:162-9.

2. Juppner H, Potts JT Jr. Immunoassays for the detection of parathyroid hormone. J Bone Miner Res. 2002; 17(Suppl 2):N81-6.

3. Slatopolsky E, Finch J, Clay P et al. A novel mechanism for skeletal resistance in uremia. Kidney Int. 2000; 58:753-61.

4. Malluche HH, Mawad H, Trueba D et al. Parathyroid hormone assays - evolution and revolutions in the care of dialysis patients. Clin Nephrol. 2003; 59:313-18.

5. Huan J, Olgaard K, Nielsen LB et al. Parathyroid hormone 7-84 induces hypocalcemia and inhibits the parathyroid hormone 1-84 secretory response to hypocalcemia in rats with intact parathyroid glands. J Am Soc Nephrol. 2006; 17:1923-30.

6. Lehmann G, Stein G, Huller $M$ et al. Specific measurement of PTH (1-84) in various forms of renal osteodystrophy (ROD) as assessed by bone histomorphometry. Kidney Int. 2005; 68:1206-14.

7. Goodman WG, Juppner H, Salusky IB, Sherrard DJ. Parathyroid hormone (PTH), PTH-derived peptides, and new PTH assays in renal osteodystrophy. Kidney Int. 2003; 63:1-11.

8. Coen G, Bonucci E, Ballanti et al. PTH 1-84 and PTH '7-84' in the noninvasive diagnosis of renal bone disease. Am J Kidney Dis. 2002; 40:348-54.

9. Monier-Faugere MC, Geng Z, Mawad H et al. Improved assessment of bone turnover by the PTH-(1-84)/large C-PTH fragments ratio in ESRD patients. Kidney Int. 2001; 60:1460-8.

10. National Kidney Foundation. K/DOQI clinical practice guidelines for bone metabolism and disease in chronic kidney disease. Am J Kidney Dis. 2003; 42(Suppl 3):1-201.

11. Souberbielle JC, Friedlander G, Cormier C. Practical considerations in PTH testing. Clin Chim Acta 2006; 366:81-9.

12. Souberbielle JC, Boutten A, Carlier MC et al. Intermethod variability in PTH measurement: Implication for the care of CKD patients. Kidney Int. 2006; 70:345-50.

13. Hsia J, Heiss G, Ren H et al. Calcium/vitamin D supplementation and cardiovascular events. Circulation 2007; 115:846-54.

14. Russo D, Miranda I, Ruocco C et al. The progression of coronary artery calcification in predialysis patients on calcium carbonate or sevelamer. Kidney Int. 2007; 72:1255-61.

15. Oliveira RB, Cancela AL, Graciolli FG et al. Early control of PTH and FGF23 in normophosphatemic CKD patients: a new target in CKD-MBD therapy? Clin J Am Soc Nephrol. 2010; 5(2):286-91.

16. Holick MF, Siris ES, Binkley N et al. Prevalence of vitamin D inadequacy among postmenopausal North American women receiving osteoporosis therapy. J Clin Endocrinol Metab. 2005; 90:3215-24.

17. Thomas MK, Lloyd-Jones DM, Thadhani RI et al. Hypovitaminosis D in medical inpatients. N Engl J Med. 1998; 338:777-83.
18. Chapuy MC, Preziosi P, Maamer M et al. Prevalence of vitamin D insufficiency in an adult normal population. Osteoporos Int. 1997; 7:439-43.

19. McKenna MJ. Differences in vitamin D status between countries in young adults and the elderly. Am J Med. 1992; 93:69-77.

20. Gordon CM, DePeter KC, Estherann G, Grace E, Emans SJ. Prevalence of vitamin D deficiency among healthy adolescents. Arch Pediatr Adolesc Med. 2004; 158:531-7.

21. Saraiva GL, Cendoroglo MS, Ramos LR et al. Prevalence of vitamin D deficiency, insufficiency and secondary hyperparathyroidism in the elderly inpatients and living in the community of the city of São Paulo, Brazil. Arq Bras Endocrinol Metabol. 2007; 51:437-42.

22. Unger MD, Cuppari L, Titan SM et al. Vitamin D status in a sunny country: where has the sun gone? Clin Nutr. 2010; 29(6):784-8. Epub 2010 Jul 15.

23. González EA, Sachdeva A, Oliver DA, Martin KJ. Vitamin D insufficiency and deficiency in chronic kidney disease. A single center observational study. Am J Nephrol. 2004; 24:503-10; Epub 2004 Sep 22.

24. Cuppari L, Garcia-Lopes MG. Hypovitaminosis D in chronic kidney disease patients: prevalence and treatment. J Ren Nutr. 2009; 19(1):38-43. Review.

25. LaClair RE, Hellman RN, Karp SL et al. Prevalence of calcidiol deficiency in CKD: a crosssectional study across latitudes in the United States. Am J Kidney Dis. 2005; 45:1026-33.

26. Saab G, Young DO, Gincherman Y, Giles K, Norwood K, Coyne DW. Prevalence of vitamin D deficiency and the safety and effectiveness of monthly ergocalciferol in hemodialysis patients. Nephron Clin Practice 2007; 105:132-8.

27. Mucsi I, Almási C, Deák G et al. Serum 25(OH)vitamin $\mathrm{D}$ levels and bone metabolism in patients on maintenance hemodialysis. Clin Nephrol. 2005; 64:288-94.

28. Shah N, Bernardini J, Piraino B. Prevalence and correction of $25(\mathrm{OH})$ vitamin $\mathrm{D}$ deficiency in peritoneal dialysis patients. Perit Dial Int. 2005;2 5:362-6.

29. Boudville NC, Hodsman AB. Renal function and 25-hydroxyvitamin D concentrations predict parathyroid hormone levels in renal transplant patients. Nephrol Dial Transplant. 2006; 21:2621-4.

30. Ooms ME, Roos JC, Bezemer PD et al. Prevention of bone loss by vitamin D supplementation in elderly women: A randomized doubleblind trial. J Clin Endocrinol Metab. 1995; 80:1052-8.

31. Khaw KT, Sneyd MJ, Compston J. Bone density, parathyroid hormone and 25-hydroxyvitamin $\mathrm{D}$ concentrations in middle aged women. Br Med J. 1992; 305:273-7.

32. Holick MF, Matsuoka LY, Wortsman J. Age, vitamin D, and solar ultraviolet. Lancet 1989; 2:1104-5.

33. Clemens TL, Adams JS, Henderson SL, Holick MF. Increased skin pigment reduces the capacity of skin to synthesise vitamin D3. Lancet 1982; 1:74-6.

34. National Kidney Foundation. K/DOQI clinical practice guidelines for bone metabolism and disease in chronic kidney disease. Am J Kidney Dis. 2003; 42(suppl.3):1-201. 
35. Zisman AL, Hristova M, Ho LT, Sprague SM. Impact of ergocalciferol treatment of vitamin D deficiency on serum parathyroid hormone concentrations in chronic kidney disease. Am J Nephrol. 2007; 27:36-43; Epub 2007 Jan 11.

36. Al-Aly Z, Qazi RA, González EA, Zeringue A, Martin KJ. Changes in serum 25-hydroxyvitamin D and plasma intact PTH levels following treatment with ergocalciferol in patients with CKD. Am J Kidney Dis. 2007; 50:59-68.

37. Barreto FC, Barreto DV, Moyses RM et al. K/DOQIrecomeend intact PTH levels do not prevent low-turnover bone diseases in hemodialysis patients. Kidney Int. 2008; 73:771-7.

38. Kalantar-Zadh K, Kuwae N, Regidor DL et al. Survival predictability of time-varying indicators of bone disease in maintenance hemodialysis patients. Kidney Int. 2006; 70:771-80.

39. Tentori F, Blayney MJ, Albert JM et al. Mortality Risk for dialysis patients with different levels of serum calcium, phosphorus, and PTH: The dialysis outcomes and practice patterns study (DOPPS). Am J Kidney Dis. 2008; 52:519-30.

40. Stevens LA, Djurdjev O, Cardew S, Cameron EC, Levin A. Calcium, Phosphate, and parathyroid hormone levels in combination and as a function of dialysis duration predict mortality. Evidence for the complexity of the association between mineral metabolism and outcomes. J Am Soc Nephrol. 2004; 15:770-9.

41. Pitts TO, Piraino BH, Mitro $\mathrm{R}$ et al. Hyperparathyroidism and 1,25-dihydroxyvitamin D deficiency in mild, moderate, and severe renal failure. J Clin Endocrinol Metab. 1988; 67:876-81.

42. Denda M, Finch J, Brown AJ, Nishii Y, Kubodera $\mathrm{N}$, Slatopolsky E. 1,25-dihydroxyvitamin D3 and 22-oxacalcitriol prevent the decrease in vitamin $\mathrm{D}$ receptor content in the parathyroid glands of uremic rats. Kidney Int. 1996; 50:34-9.

43. Fukuda N, Tanaka H, Tominaga Y, Fukagawa M, Kurokawa K, Seino Y. Decreased 1,25-dihydroxyvitamin D3 receptor density is associated with a more severe form of parathyroid hyperplasia in chronic uremic patients. J Clin Invest. 1993; 92:1436-43.

44. Baker LR, Muir JW, Sharman VL et al. Controlled trial of calcitriol in hemodialysis patients. Clin Nephrol. 1986; 26:185-91.

45. Gallieni M, Brancaccio D, Padovese P et al.Low-dose intravenous calcitriol treatment of secondary hyperparathyroidism in hemodialysis patients. Italian Group for the Study of Intravenous Calcitriol. Kidney Int. 1992; 42:1191-8.

46. Goodman WG, Ramirez JÁ, Belin TR et al. Development of adynamic boné in patients with secondary hyperparathyroidism after intermittent calcitriol therapy. Kidney Int. 1994; 46:1160-6.

47. Malluche HH, Mawad H, Koszewski NJ. Update on vitamin $\mathrm{D}$ and its newer analogues: actions and rationale for treatment in chronic renal failure. Kidney Int. 2002; 62:367-74.

48. Liou HH, Chiang SS, Huang TP, Shieh SD, Akmal M. Comparative effect of oral or intravenous calcitriol on secondary hyperparathyroidism in chronic hemodialysis patients. Miner Eletrolyte Metab. 1994; 20:97-102.
49. Borazan A, Ustun H, Cefle A, Seritmez N, Yilmaz A. Comparative efficacy of oral and intravenous calcitriol treatment in haemodialysis patients: Effects on serum biochemistry and cytokine levels. J Int Med Res. 2003; 31:489-96.

50. Moe SM, Kraus MA, Gassensmith CM, Fineberg NS, Gannon FH, Peacock M. Safety and efficacy of pulse and daily calcitriol in patients on CAPD: a randomized trial. Nephrol Dial Transplant. 1998; 13:1234-41.

51. Herrmann P, Ritz E, Schmidt-Gayk $\mathrm{H}$ et al. Comparison of intermittent and continuous oral administration of calcitriol in dialysis patients: A randomized prospective trial. Nephron. 1994; 67:48-53.

52. Indridason OS, Quarles LD, for the Durham Renal Osteodystrophy Study Group. Comparison of treatments for mild secondary hyperparathyroidism in hemodialysis patients. Kidney Int. 2000; 57:282-92.

53. Fischer ER, Harris DC. Comparison of intermittent oral and intravenous calcitriol in hemodialysis patients with secondary hyperparathyroidism. Clin Nephrol. 1993; 40:216-20.

54. Quarles LD, Yohay DA, Carroll BA et al. Prospective trial of pulse oral versus intravenous calcitriol treatment of hyperparathyroidism in ESRD. Kidney Int. 1994; 45:1710-21.

55. Slatopolsky E, Weerts C, Thielan J, Horst R, Harter $\mathrm{H}$, Martin KJ. Marked suppression of secondary hyperparathyroidism by intravenous administration of 1,25-dihydroxy-cholecalciferol in uremic patients. J Clin Invest. 1984; 74:2136-43.

56. Bacchini G, Fabrizi F, Pontoriero G, Marcelli D, Di Filippo S, Locatelli F. "Pulse oral" versus intravenous calcitriol therapy in chronic hemodialysis patients. A prospective and randomized study. Nephron. 1997; 77:267-72.

57. Levine BS, Song M. Pharmacokinetics and efficacy of pulse oral versus intravenous calcitriol in hemodialysis patients. J Am Soc Nephrol. 1996; 7:488-96.

58. Mazess RB, Elangovan L. A review of intravenous versus oral vitamin D hormone therapy in hemodialysis patients. Clin Nephrol. 2003; 59:319-25.

59. Andress DL, Norris KC, Coburn JW, Slatopolsky EA, Sherrard DJ. Intravenous calcitriol in the treatment of refractory osteitis fibrosa of chronic renal failure. N Engl J Med. 1989; 321:274-9.

60. LLach F, Hervas J, Cerezo S. The importance of dosing intravenous calcitriol in dialysis patients with severe hyperparathyroidism. Am J Kidney Dis. 1995; 26:845-51.

61. Rodriguez-Garcia M, Fernandez-Martin JL, Castañeda JR, Hervás-Sánchez J, Cannata-Andía JB, and the Caldial Study Group. Advantages of adjusting the initial dose of intravenous calcitriol according to PTH levels. Kidney Int. 2003; 63(suppl 85):S79-S82.

62. Morosetti M, Jankovic L, Cetani F et al. High doses of calcitriol in the treatment of severe secondary hyperparathyroidism. J Nephrol. 2004; 17:95-100.

63. Malberti F, Corradi B, Cosci P, Calliada F, Marcelli D, Imbasciati E. Long-term effects of intravenous calcitriol therapy on the control of secondary hyperparathyroidism. Am J Kidney Dis. 1996; 28:704-12. 
64. Costa AFP, Reis LM, Ribeiro MC, Moysés RMA, Jorgetti V. Effects of calcitriol on parathyroid function and on bone remodelling in secondary hyperparathyroidism. Nephrol Dial Transplant. 2003; 18:743-9.

65. Katoh N, Nakayama M, Shigematsu T et al. Presence of sonographically detectable parathyroid glands can predict resistance to oral pulsed-dose calcitriol treatment of secondary hyperparathyroidism. Am J Kidney Dis. 2000; 35:465-8.

66. Sprague SM, Llach F, Amdahl M et al. Paricalcitol versus calcitriolin the treatment of secondary hyperparathyroidism. Kidney Int. 2003; 63:1483-90.
67. Moe SM, Chertow GM, Quarles LD, Goodman WG, Block GA, Drueke TB. Achieving NKF-K/DOQI ${ }^{\mathrm{TM}}$ bone metabolism and disease treatment goals with cinacalcet HCL. Kidney Int. 2005; 67:760-71.

68. Tominaga Y, Tanaka Y, Sato K, Nagasaka T, Takagi H. Histopathology, pathophysiology, and indications for surgical treatment of renal hyperparathyroidism. Semin Surg Oncol. 1997 Mar-Apr;13(2):78-86. 